\title{
Le chemin du médicament dans les maladies neuromusculaires : une nouvelle aventure
}

L e chemin du médicament, dessiné par les dirigeants de l'AFM-Téléthon dès la fin des années 1980 a été le fondement de la stratégie de notre Association. Étape après étape, avec détermination et conviction, de nombreux obstacles ont été franchis.

Grâce aux progrès de la biologie moléculaire, il est maintenant possible d'agir en amont, dès l'origine de la maladie neuromusculaire, en corrigeant l'anomalie génétique. La déficience d'une protéine manquante ou anormale pourra ainsi être palliée par un médicament issu de la pharmacologie, de la thérapie cellulaire ou bien de la thérapie génique.

Aujourd'hui, la dernière partie de ce chemin, celle qui va du développement à la mise sur le marché du traitement, est largement amorcée pour bon nombre de maladies. Mais les défis sont multiples, qu'il s'agisse de bioproduction, de mesure de l'efficacité des traitements, des discussions avec les agences réglementaires ou bien d'autres encore...

Toujours fidèle à ses valeurs et à son engagement, l'AFM-Téléthon veut garantir, à toute personne atteinte de maladie neuromusculaire, l'accès aux traitements innovants, avec une mise sur le marché à un prix juste et mâ̂trisé. Les nouvelles générations doivent enfin connaitre le sens du mot GUÉRIR.

Dans le domaine des maladies rares, dont font partie les maladies neuromusculaires, il reste encore de nombreux besoins non satisfaits. Dans l'Union Européenne (UE), l'homologation des médicaments orphelins, l'assistance au protocole et l'autorisation de commercialisation s'inscrivent dans une procédure centralisée. La détermination du prix d'un produit innovant et son remboursement, quant à eux, sont laissés à l'appréciation de chaque État membre. Ces évaluations nationales sont indépendantes les unes des autres et ne sont pas reliées au processus réglementaire centralisé de l'Agence européenne du Médicament (EMA).

Les médicaments innovants neuromusculaires actuellement disponibles ou en passe de l'être sont encore trop peu nombreux.

Depuis 2006, la voie a été tracée par l'enzyme recombinante $\left(\right.$ Myozyme ${ }^{\circledR}$ ) prescrite dans la glycogénose musculaire de type II (maladie de Pompe). Son prix est de $300000 € /$ patient/an.

Pour le Spinraza ${ }^{\circledR}$ (ou nusinersen), indiqué dans toutes les formes d'amyotrophies spinales (SMA) aux États-Unis, son prix est de 125000 USD par ampoule, soit pour une moyenne de 750000 USD la première année, et la moitié les années suivantes. Dans l'Union Européenne ce produit a obtenu, le 21 avril dernier, un avis technique positif du Comité des Médicaments à Usage Humain (CHMP) lequel dépend de l'Agence Européenne du Médicament. Bien que l'avis du CHMP ne soit que consultatif, il est très rare que la Commission européenne ne le suive pas. Elle a désormais deux mois pour décider de valider ou non l'autorisation de mise sur le marché européenne (AMM) ${ }^{1}$.

Une bonne médication pour les patients atteints de maladie rare doit être une médication à la fois accessible et disponible dans leur pays. Il convient donc de concilier, d'une part, un accès plus large aux médicaments orphelins et, de l'autre, la stabilité des systèmes de santé. Tel doit donc être notre objectif commun: des traitements plus nombreux, plus efficaces, moins coûteux, mais aussi plus rapidement disponibles.

Inscrite pleinement dans le sens de l'histoire de l'AFM-Téléthon, une nouvelle aventure se profile.

Il est donc nécessaire de veiller à ce que les processus d'évaluation de la valeur, de la tarification et du financement reflètent les spécificités des maladies rares et contribuent à la durabilité des systèmes de santé et du développement de l'innovation.

Dans cette ultime étape qui doit transformer les candidatsmédicaments en traitements efficaces et accessibles à tous, plus que jamais nous devons faire preuve de détermination. Pour nos enfants, pour nos proches et pour la génération à venir, nous nous devons de réussir !

The new frontier in neuromuscular diseases therapy: clearing the path from drug discovery to clinics

Laurence Tiennot-Herment Présidente de l'AFM-Téléthon

LIENS D'INTÉRÊT

L'auteur déclare n'avoir aucun lien d'intérêt concernant les données publiées dans cet article.

\section{NOTE}

${ }^{1}$ NDLR : au moment de la mise sous presse, Biogen a annoncé le 01/06/17 avoir obtenu une autorisation de mise sur le marché (AMM) en Europe.

http://media.biogen.com/press-release/investor-relations/spinraza-nusinersen-approved-european-union-first-treatment-spinal- 